\title{
Choice of Countermeasures in Project Risk Management Using Fuzzy Modelling
}

\author{
D. Kuchta, D. Skorupka
}

\author{
Dorota Kuchta* \\ 1. Wroclaw University of Technology \\ Poland, 50-370 Wroclaw, Wybrzeze Wyspianskiego 27, \\ dorota.kuchta@pwr.wroc.pl \\ 2. Tadeusz Kosciuszko Military Academy of Land Forces \\ Poland, 51-150 Wroclaw, ul. Czajkowskiego 109, \\ *Corresponding author

\section{Dariusz Skorupka} \\ Tadeusz Kosciuszko Military Academy of Land Forces \\ Poland, Wroclaw
}

\begin{abstract}
The paper proposes a new method for project risk management. It is proposed how, after risk identification, the countermeasures for risk mitigation and elimination can be selected, taking into account the cost and effort linked to them as well as the weights assigned by the decision maker to risk attributes, such as probability or consequences, and the values of those attributes. The risk attributes and weights, as well as the maximal total risk and the maximal total effort of risk mitigation accepted by the decision maker for the project are expressed as fuzzy numbers, which in turn constitute models for linguistic expressions.

Keywords: project risk, risk mitigation, risk transfer, risk elimination, fuzzy logicbased optimisation.
\end{abstract}

\section{Introduction}

Project risk management is a very important subject and there is a lot of literature connected to it. There are also a lot of risk definitions (it is assumed here, after [1], that risk is defined as an event which may happen and if it does, it will have negative consequences on at least one of project success determinants ${ }^{1}$ ) and risk management methods or techniques (e.g. [1]). Most of them can be summarized as follows:

I. Risk identification;

II. Risk evaluation - the risks identified in point I. are evaluated on the basis of their attributes (like probability and consequences);

III. Risk elimination, transfer or mitigation - taking some steps or countermeasures which eliminate some risks or decrease the evaluation of others;

IV. Risk control during the project execution;

V. Lesson learned recording.

The problem to which this paper wants to contribute is point III. of the above procedure. There are no formal methods in the literature, apart from one presented in [10], helping the decision maker in this step. Normally only informal procedures are proposed (e.g. [6]). And in [10] the authors assume among others that the probability of the risks cannot be explicitly

\footnotetext{
${ }^{1}$ Project success determinants are discussed later in the paper.
} 
influenced by the countermeasures, but only the expected value of monetary losses, which in our opinion is wrong - in reality usually each risk attribute (e.g. probability, consequences, etc.), can be individually influenced by individual countermeasures. The authors of [10] assume also that the goal is the maximization of the monetary expected value of risk reduced by the countermeasures, whereas we assume that the goal should be the minimization of countermeasures total cost needed to attain a fixed total risk level of the project, which we think to be more realistic. Also, in [10] crisp values are required for risk attributes, ever for quality, whereas we assume linguistic expressions (fuzzy numbers) for risk attributes, like it is done e.g. in [4] and [9], as we think crisp values would usually be difficult to obtain from the decision maker. Also, basing ourselves on [9], it is assumed here that different risk attributes may have various weights in the eyes in the decision maker, which in [10] is ignored. For one decision maker mostly the consequences may count (this is the case for projects where consequences are injuries or deaths of human beings), for some decision makers mostly the probabilities (it is so for many public projects, where each problem, even a relatively small one, is noticed by the media who make a big deal of it). What is more, nowhere in the literature the notion of risk levels (e.g. project level, closest environment level, further environment level, national level, international level etc., introduced in [8]) and the effort linked to an effective application of countermeasures on each of the different levels are combined with the choice of risk countermeasures, and this is done in the present paper.

\section{Basic notions and notation}

As it was mentioned above, project risk is an event which might happen and if it happens, it will have negative consequences on one of the success determinants of the project. There are several determinants of project success (for a review see e.g. [2]), like time, quality, cost, customer satisfaction, etc. Let us denote those determinants as $D_{l}, l=1, \ldots, L$.

The risks may concern several levels ( [8]), e.g. the project level, the closer environment level (for construction projects this would be the construction market level), the further environment level (for construction projects this would be the national level), etc. The various levels are denoted as $L_{t}, t=1, \ldots, T$. The higher the level index, the harder accessible and the less possible to influence the level is, thus the less manageable the risks linked to this level are.

It is assumed that (point I. of the project risk management procedure given above) $P_{t}^{l}$ risks for each level $L_{t}, t=1, \ldots, T$ and for each determinant $D_{l}, l=1, \ldots, L$ have been identified (the risks will be denoted as $R_{k}^{t, l}, k=1, \ldots, P_{t}^{l}$ ). Each risk $R_{k}^{t, l}$, if it happens, it concerns level $L_{t}$ (thus, if one wanted to mitigate or eliminate it, one would have to act on the respective level) and will influence determinant $D_{l}, l=1, \ldots, L$ of project success. It is also assumed that each risk is linked to exactly one level and to exactly one determinant, but this assumption would not be difficult to be given up.

Each risk $R_{k}^{t, l}$ will have several attributes. Usually two or at the most three risk attributes are considered: the probability of occurrence, the consequences of the occurrence (for the project success determinant linked to the specific risk, i.e. risks may have consequences for time, cost, quality, customer satisfaction etc.) and sometimes the difficulty in an early detection or in early forecasting of the risk occurrence (e.g. [6]). Sometimes additional attributes may be important, like the probability of the occurrence of consequences once the risk has happened, the degree of influence we have on the occurrence of the risk etc. The latter attribute is especially important in case we have to consider several levels on which the risks may occur: the further the level, the less effective our countermeasures may be.

Let us denote the number of attributes as $A$, and the attributes themselves as $v_{a}, a=1, \ldots, A$. The attributes are functions of risks, thus the value of the $a$-th attribute of risk $R_{k}^{t, l}$ will be 
$v_{a}\left(R_{k}^{t, l}\right), a=1, \ldots, A, l=1, \ldots, L, t=1, \ldots, T, k=1, \ldots, P_{t}^{l} . v_{a}\left(R_{k}^{t, l}\right)$ will be all measured in the same scale, their values will be given by experts.

As mentioned above, various risk attributes may have various weights in the eyes of the decision maker. Each attribute $v_{a}\left(R_{k}^{t, l}\right)$ will be thus assigned a weight $w_{a}\left(R_{k}^{t, l}\right)$, also according to a given scale.

Finally, it is possible to use the defined parameters to evaluate each risk (point II. of the project risk management procedure). The evaluation $R E V\left(R_{k}^{t, l}\right)$ of each risk may be defined using one of the formulae proposed e.g. in $[6]^{2}$, e.g. the following one:

$$
R E V\left(R_{k}^{t, l}\right)=\frac{\sum_{a=1}^{A} w_{a}\left(R_{k}^{t, l}\right) \cdot v_{a}\left(R_{k}^{t, l}\right)}{\sum_{a=1}^{A} w_{a}\left(R_{k}^{t, l}\right)}
$$

As a further step (point III. of the project risk management procedure), it is proposed to try to identify certain countermeasures to eliminate or mitigate the risks. It is assumed that each measure may concern only one level, one project success determinant and one risk attribute. This assumption seems natural: one countermeasure can e.g. be effective only on the national level and affect only the time, i.e. a possible delay being the consequence of a certain risk, another countermeasure may concern only the project level and affect the cost and the probability of an event which would increase cost. Let us introduce also a simplifying assumption that each attribute of each risk can be affected by only one measure. Thus, for each $l=1, \ldots, L, t=$ $1, \ldots, T$ and $a=1, \ldots, A$ let us define a set of measures $\left(M_{h}^{t, l, a}, h=1, \ldots, H_{t}^{l, a}\right)$. Then, for each attribute $v_{a}\left(R_{k}^{t, l}\right), a=1, \ldots, A$ of each risk $R_{k}^{t, l}\left(k=1, \ldots, P_{t}^{l}\right)$ it is identified which measure among the measures $\left\{M_{h}^{t, l, a}, h=1, \ldots, H_{t}^{l, a}\right\}$ can be applied, and the index of this measure will be denoted as $M\left(v_{a}\left(R_{k}^{t, l}\right)\right)$. The value of each attribute of the risk $R_{k}^{t, l}$ after the application of the measure indexed by $M\left(v_{a}\left(R_{k}^{t, l}\right)\right)$ will be equal to $m\left(v_{a}\left(R_{k}^{t, l}\right)\right)$. Of course it is true $m\left(v_{a}\left(R_{k}^{t, l}\right)\right)<$ $v_{a}\left(R_{k}^{t, l}\right)$. For some risks and attributes no countermeasure may exist - then it is set everywhere $m\left(v_{a}\left(R_{k}^{t, l}\right)\right)=v_{a}\left(R_{k}^{t, l}\right)$.

The evaluation of each risk after the application of the measures will be

$$
R E V\left(R_{k}^{t, l}\right)=\frac{\sum_{a=1}^{A} w_{a}\left(R_{k}^{t, l}\right) \cdot m\left(v_{a}\left(R_{k}^{t, l}\right)\right)}{\sum_{a=1}^{A} w_{a}\left(R_{k}^{t, l}\right)}
$$

Each measure will of course be linked to a cost, measured in monetary values, denoted as $C\left(M_{h}^{t, l, a}\right), h=1, \ldots, H_{t}^{l}, l=1, \ldots, L, t=1, \ldots, T, a=1, \ldots, A$. Apart from the cost, the implementation of each measure will mean an effort, problems of various kind, the necessity to seek the access to certain people, to ask for permissions, uncertainty as far the effect of the measure is concerned, etc. - usually the bigger, the higher the level index is. Thus it is assumed that the effort depends on the level, not on the individual measures (again, it is only a simplifying assumption). It will be denoted as $E_{t}, t=1, \ldots, T$ and evaluated by an expert in a fixed scale. Each single measure $M_{h}^{t, l, a}, h=1, \ldots, H_{t}^{l}, l=1, \ldots, L, a=1, \ldots, A$ will be thus linked to effort $E_{t}$.

\footnotetext{
${ }^{2}$ In [6] there is a discussion showing that from the practical point of view all the formulae are in fact equivalent.
} 


\section{Model optimising the choice of countermeasures in project risk management}

In the model we assume that the project manager wants, if possible, to achieve a small project overall risk level at the minimal cost. Also, the effort linked to the measures should be taken into account. Thus in fact three objective functions should be present in the model: the overall risk level, the cost of applying risk mitigation or elimination measures and the overall effort of applying those measures.

In order to solve this multicriteria problem, let us choose the easiest approach, where the level of all but one criteria has to be fixed, so that only one criteria formally remains as an objective function. In our opinion in most cases the decision maker will have to maintain a certain level of overall risk, and this level would be fixed beforehand. The decision maker would thus seek a way to achieve this level at the minimal cost. Also, he would like to minimize the effort, but probably rather as a secondary goal, trying to control it and be aware of it than to search for an absolute minimum. That is why the following model is proposed, but of course other formulations of this multicriteria problem would be possible too.

$$
\begin{gathered}
\sum_{\substack{h=1, \ldots, H_{t}^{l}, l=1, \ldots, L, t=1, \ldots, T, a=1, \ldots, A}} C\left(M_{h}^{t, l, a}\right) \cdot x_{h}^{t, l, a} \rightarrow \min \\
\sum_{\substack{l=1, \ldots, L, t=1, \ldots, T, a=1, \ldots, A, k=1, \ldots, P_{t}^{l}}} \frac{\sum_{a=1}^{A} w_{a}\left(R_{k}^{t, l}\right) \cdot\left(1-y_{k, a}^{t, l}\right) \cdot v_{a}\left(R_{k}^{t, l}\right)+\sum_{a=1}^{A} w_{a}\left(R_{k}^{t, l}\right) \cdot y_{k, a}^{t, l} \cdot m\left(v_{a}\left(R_{k}^{t, l}\right)\right)}{\sum_{k=\left\{1, \ldots, P_{t}^{l}\right\}}^{A} w_{a}\left(R_{k}^{t, l}\right)} \leq R L \\
\sum_{a=1} y_{k, a}^{t, l} \leq M \cdot x_{h}^{t, l, a} \\
k\left(v_{a}\left(R_{k}^{t, l}\right)\right)=h
\end{gathered}
$$

for each $h=1, \ldots, H_{t}^{l}, l=1, \ldots, L, t=1, \ldots, T, a=1, \ldots, A$

$$
\sum_{\substack{h=1, \ldots, H_{t}^{l}, l=1, \ldots, L, t=1, \ldots, T, a=1, \ldots, A}} E\left(M_{h}^{t, l, a}\right) \cdot x_{h}^{t, l, a} \leq E L
$$

where $x_{h}^{t, l, a}, h=1, \ldots, H_{t}^{l}, l=1, \ldots, L, t=1, \ldots, T, a=1, \ldots, A$, will be equal to 1 if the corresponding measure should be applied and to 0 otherwise, $y_{k, a}^{t, l}, l=1, \ldots, L, t=1, \ldots, T, a=$ $1, \ldots, A, k=1, \ldots, P_{t}^{l}$ will be binary variables fulfilling the constraint $y_{k, a}^{t, l}=0$ (thus, being able to be eliminated from the model) if the value of the $a$-th attribute of risk $R_{k}^{t, l}$ would not be changed thanks to the corresponding countermeasure. The constant $R L$ stands for the admissible overall project risk level, the constant $E L$ for the admissible effort level linked to the selected countermeasures and the constant $M$ for a sufficiently big number.

Actually, in practice most of the parameters in the above model may be impossible to determine in an exact way and may require a fuzzy formulation. Because of the limited scope of the paper, we assume, basing ourselves on $[4,5,7,9]$, only the risk attributes and the weights of the attributes to be fuzzy, as well the constants $R L$ and $E L$ and cost values will be crisp.

For the fuzzy modeling, let us use here the language from [9], where the authors consider three risk attributes (probability of occurrence, severity of consequences, early detection difficulty) whose values can take five fuzzy vales each ( VH - very high, $\mathrm{H}$ - high, $\mathrm{M}$ - moderate, $\mathrm{L}$ 
- low, VL - very low). The same 5 values are assigned in [9] to the attributes weights. The human language assumed in [9] at the background is as follows $\left(\widetilde{A}=\left(a_{1}, a_{2}, a_{3}\right)\right.$ stands for the triangular fuzzy number with support $\left[a_{1}, a_{3}\right]$ and $a_{2}$ as the value with the membership degree 1$)$ :

Table 1: Linguistic scale for risk attributes, their weights and the effort linked to the countermeasures [9]

\begin{tabular}{|c|c|}
\hline Linguistic term & Corresponding triangular fuzzy number \\
\hline Very low (VL) & $(0,0,0.25)$ \\
\hline Low (L) & $(0,0.25,0.5)$ \\
\hline Moderate (M) & $(0,0.5,0.75)$ \\
\hline High & $(0,0.75,1)$ \\
\hline Very High (VH) & $(0.75,1,1)$ \\
\hline
\end{tabular}

For $R L$ and $E L$ we will use fuzzy numbers $\widetilde{A}=\left(a_{1}, a_{2}, a_{3}\right)$ with $a_{2}=a_{3}$. The decision maker might be supported in the choice of the fuzzy numbers for $R L$ and $E L$ by means of questions similar to the following ones:

- For $R L$ : how many serious risks at the maximum would he accept, and how many serious risk he thinks have to be linked to each project at the minimum (as there are no projects without risk and accepting risks opens new possibilities)?

- For EL: similar questions, but regarding difficult countermeasures.

With fuzzy parameters denoted by $\sim$, we would have a mathematical programming problem with fuzzy parameters in constraint (4), which would become

$$
\sum_{\substack{l=1, \ldots, L, t=1, \ldots, T, a=1, \ldots, A, k=1, \ldots, P_{t}^{l}}} \frac{\sum_{a=1}^{A} \widetilde{w}_{a}\left(R_{k}^{t, l}\right) \cdot\left(1-y_{k, a}^{t, l}\right) \cdot \widetilde{v}_{a}\left(R_{k}^{t, l}\right)+\sum_{a=1}^{A} \widetilde{w}_{a}\left(R_{k}^{t, l}\right) \cdot y_{k, a}^{t, l} \cdot \widetilde{m}\left(v_{a}\left(R_{k}^{t, l}\right)\right)}{\sum_{a=1}^{A} \widetilde{w}_{a}\left(R_{k}^{t, l}\right)} \leq \widetilde{R L}
$$

The rest of model (3) - (6) will remain unchanged.

Constraint (7) is not unequivocal. It may be interpreted in many ways, according to the way the decision maker chooses to compare fuzzy numbers. Also, the fuzzy numbers on the left hand side have to be multiplied with a constant and added one to another.

Let us thus discuss shortly basic arithmetical operations on fuzzy numbers and the simplest approaches of comparing them. Let us thus consider two triangular fuzzy numbers $\widetilde{A}$ and $\widetilde{B}$, based, respectively, on the following triples of crisp numbers: $\left(a_{1}, a_{2}, a_{3}\right)$ and $\left(b_{1}, b_{2}, b_{3}\right)$. Then we have:

- $\widetilde{A}+\widetilde{B}=\left(a_{1}+b_{1}, a_{2}+b_{2}, a_{3}+b_{3}\right)$

- $\widetilde{A} \cdot \widetilde{B}=\left(a_{1} b_{1}, a_{2} b_{2}, a_{3} b_{3}\right)$ if all the parameters are positive

- For each crisp number $r$ we have: $r \cdot \widetilde{A}=\widetilde{A} \cdot r=\left(r a_{1}, r a_{2}, r a_{3}\right)$

If it comes to the inequality $\widetilde{A} \leq \widetilde{B}$, it may be interpreted depending on the pessimism/optimism degree of the decision maker. E.g. three interpretations listed below are possible, where the first one is the most pessimistic (it is most difficult for $\widetilde{A}$ to satisfy it) and the last one the most optimistic: 
- $a_{3} \leq b_{1}$

- $\frac{a_{3}+a_{2}}{2} \leq \frac{b_{2}+b_{1}}{2}$

- $a_{2} \leq b_{2}$

- $\frac{a_{2}+a_{1}}{2} \leq \frac{b_{3}+b_{2}}{2}$

- $a_{1} \leq b_{3}$

For other possibilities see e.g. [3]. Choosing (in cooperation with the decision maker) one of the approaches, we can turn constraint (7) into a crisp constraint.

\section{Example}

Let us consider a real world construction project, presented in detail in [8]. There are over 30 risks there and a similar number of countermeasures. Here let us consider only a selection of the data. Thus we have the following risks:

- on the project level, determinant time: $R_{1}^{1,1}$ - erroneous identification of soil;

- on the project level, determinant cost: $R_{1}^{1,2}$ - accidents during the construction;

- on the construction market level, determinant time: $R_{1}^{2,1}$ - non-availability of work force at the budgeted cost;

- on the construction market level, determinant cost: $R_{1}^{2,2}$ - non-availability of required equipment;

- on the national level, determinant cost: $R_{1}^{3,2}$ - unfavorable inflation.

The values of the risk attributes were assessed by the experts, as well as the weights of the attributes (we assume in the example that the weights are the same for all the risks).

Table 2: Risks attributes and their weights in the example

\begin{tabular}{|l|l|l|l|l|l|l|}
\hline Risk & $\mathbf{R}_{\mathbf{1}}^{\mathbf{1}, \mathbf{1}}$ & $\mathbf{R}_{\mathbf{1}}^{\mathbf{1 , 2}}$ & $\mathbf{R}_{\mathbf{1}}^{\mathbf{2 , 1}}$ & $\mathbf{R}_{\mathbf{1}}^{\mathbf{2 , 2}}$ & $\mathbf{R}_{\mathbf{1}}^{\mathbf{3} \mathbf{2}}$ & $\begin{array}{l}\text { Weight of } \\
\text { the attribute }\end{array}$ \\
\hline Probability of occurrence & $\mathrm{M}$ & $\mathrm{VH}$ & $\mathrm{M}$ & $\mathrm{H}$ & $\mathrm{H}$ & $\mathrm{L}$ \\
\hline Consequences of occurrence & $\mathrm{H}$ & $\mathrm{VH}$ & $\mathrm{H}$ & $\mathrm{M}$ & $\mathrm{VH}$ & $\mathrm{VH}$ \\
\hline Difficulty in early detection & $\mathrm{VH}$ & $\mathrm{H}$ & $\mathrm{L}$ & $\mathrm{L}$ & $\mathrm{L}$ & $\mathrm{M}$ \\
\hline $\begin{array}{l}\text { Difficulty in influencing the risk } \\
\text { by countermeasures }\end{array}$ & $\mathrm{L}$ & $\mathrm{L}$ & $\mathrm{H}$ & $\mathrm{H}$ & $\mathrm{VH}$ & $\mathrm{M}$ \\
\hline
\end{tabular}

Five countermeasures have been identified, together with their cost and effort: 
Table 3: Countermeasures, their cost, efforts and effects for the example

\begin{tabular}{|l|l|l|l|l|}
\hline Countermeasure & $\begin{array}{l}\text { Risk and } \\
\text { attribute } \\
\text { influenced }\end{array}$ & $\begin{array}{l}\text { Effect on } \\
\text { the at- } \\
\text { tribute }\end{array}$ & $\begin{array}{l}\text { Cost of the coun- } \\
\text { termeasure } \begin{array}{l}\text { (in } \\
\text { monetary units } \\
\text { denoted } \$ \text { ) }\end{array}\end{array}$ & $\begin{array}{l}\text { Effort linked } \\
\text { to the coun- } \\
\text { termeasure }\end{array}$ \\
\hline $\begin{array}{l}\text { Having ready an alter- } \\
\text { native technology which } \\
\text { can be used immediately } \\
\text { when the soil problem is } \\
\text { detected, } M_{1}^{1,1,2}\end{array}$ & $\begin{array}{l}R_{1}^{1,1} \text { conse- } \\
\text { quences }\end{array}$ & $\mathrm{H} \rightarrow \mathrm{L}$ & $40 \$$ & $\mathrm{~L}$ \\
\hline $\begin{array}{l}\text { Additional check of all } \\
\text { the equipment and work- } \\
\text { ers training, } M_{1}^{1,2,1}\end{array}$ & $\begin{array}{l}R_{1}^{1,2}, \text { proba- } \\
\text { bility }\end{array}$ & $\mathrm{VH} \rightarrow \mathrm{H}$ & $30 \$$ & $\mathrm{~L}$ \\
\hline $\begin{array}{l}\text { Training offered to pupils } \\
\text { which are about to grad- } \\
\text { uate a construction high } \\
\text { school, } M_{1}^{2,1,2}\end{array}$ & $\begin{array}{l}R_{1}^{2,1}, \text { proba- } \\
\text { bility }\end{array}$ & $\mathrm{M} \rightarrow \mathrm{L}$ & $50 \$$ & $\mathrm{H}$ \\
\hline $\begin{array}{l}\text { Renting or reserving } \\
\text { the needed equipment } \\
\text { already now, } M_{1}^{2,2,1}\end{array}$ & $\begin{array}{l}R_{1}^{2,2}, \text { conse- } \\
\text { quences }\end{array}$ & $\mathrm{M} \rightarrow \mathrm{VL}$ & $40 \$$ & $\mathrm{H}$ \\
\hline $\begin{array}{l}\text { Using a more stable cur- } \\
\text { rency in all the transac- } \\
\text { tions, } M_{1}^{3,2,1}\end{array}$ & $\begin{array}{l}R_{1}^{3,2}, \text { proba- } \\
\text { bility }\end{array}$ & $\mathrm{H} \rightarrow \mathrm{L}$ & $20 \$$ & $\mathrm{VH}$ \\
\hline
\end{tabular}

For the example we have:

- $l=1,2$ (we consider two project success determinants: time and cost)

- $t=1,2,3$ (we have three levels: the project level, the construction market level, the national level);

- $a=1,2,3,4$ (we have four risk attributes corresponding to the rows of Table 2);

- $H_{t}^{l, a}=1$ for the following triples $(t, l, a)$, which can be read off from the rows of Table 3: $(1,1,2),(1,2,1),(2,1,1),(2,2,2),(3,2,1)$. Otherwise it is equal to 0 ;

- decision variables (binary) are as follows: $x_{1}^{1,1,2}, y_{1,2}^{1,1}, x_{1}^{1,2,1}, y_{1,1}^{1,2}, x_{1}^{2,1,2}, y_{1,1}^{2,1}, x_{1}^{2,2,2}, y_{1,2}^{2,2}$, $x_{1}^{3,2,2}, y_{1,1}^{3,2}$.

Let us assume $R L=E L=(2,3,3)$. We get then the following model (using values from Table 1,2 and 3 and formulae (8)-(10)):

$$
40 x_{1}^{1,1,2}+30 x_{1}^{1,2,1}+50 x_{1}^{2,1,2}+40 x_{1}^{2,2,2}+20 x_{1}^{3,2,2} \rightarrow \min
$$




$$
\begin{aligned}
A= & (0,75,1,1)\left(1-y_{1,2}^{1,1}\right)(0,0,75,1)+(0,75,1,1) y_{1,2}^{1,1}(0,0,25,0,5) \\
& +(0,0,25,0,5)\left(1-y_{1,1}^{1,2}\right)(0,75,1,1)+(0,0,25,0,5) y_{1,1}^{1,2}(0,0,75,1) \\
& +(0,0,25,0,5)\left(1-y_{1,1}^{2,1}\right)(0,0,5,0,75)+(0,0,25,0,5) y_{1,1}^{2,1}(0,0,25,0,5) \\
& +(0,75,1,1)\left(1-y_{1,2}^{2,2}\right)(0,0,5,0,75)+(0,75,1,1) y_{1,2}^{2,2}(0,0,0,25) \\
& +(0,0,25,0,5)\left(1-y_{1,1}^{3,2}\right)(0,0,75,1)+(0,0,25,0,5) y_{1,1}^{3,2}(0,0,25,0,5) \\
= & (0,0,75,1)\left(1-y_{1,2}^{1,1}\right)+(0,0,25,0,5) y_{1,2}^{1,1}+(0,0,25,0,5)\left(1-y_{1,1}^{1,2}\right) \\
& +(0,0,1875,0,5) y_{1,1}^{1,2}+(0,0,125,0,375)\left(1-y_{1,1}^{2,1}\right)+(0,0,0625,0,25) y_{1,1}^{2,1} \\
& +(0,0,5,0,75)\left(1-y_{1,2}^{2,2}\right)+(0,0,0,25) y_{1,2}^{2,2}+(0,0,1875,0,5)\left(1-y_{1,1}^{3,2}\right) \\
& +(0,0,0625,0,25) y_{1,1}^{3,2}
\end{aligned}
$$

$$
\begin{gathered}
B=(0,75,1,1)+(0,0,25,0,5)+(0,0,25,0,5)+(0,75,1,1)+(0,0,25,0,5)=(1,5,2,75,3,5) \\
\frac{A}{B} \leq(2,3,3) \\
y_{1,2}^{1,1} \leq M x_{1}^{1,1,2}, y_{1,1}^{1,2} \leq M x_{1}^{1,2,1}, y_{1,1}^{2,1} \leq M x_{1}^{2,1,2}, y_{1,2}^{2,2} \leq M x_{1}^{2,2,2}, y_{1,1}^{3,2} \leq M x_{1}^{3,2,2} \\
(0,0,25,0,5) x_{1}^{1,1,2}+(0,0,25,0,5) x_{1}^{1,2,1}+(0,0,75,1) x_{1}^{2,1,2} \\
+(0,0,75,1) x_{1}^{2,2,2}+(0,75,1,1) x_{1}^{3,2,2} \leq(2,3,3)
\end{gathered}
$$

Assuming the method (12) of comparing fuzzy numbers and combining (17), (18) i (19), we get the following crisp linear programming model:

$$
\begin{gathered}
40 x_{1}^{1,1,2}+30 x_{1}^{1,2,1}+50 x_{1}^{2,1,2}+40 x_{1}^{2,2,2}+20 x_{1}^{3,2,2} \rightarrow \min \\
0,875\left(1-y_{1,2}^{1,1}\right)+0,375 y_{1,2}^{1,1}+0,375\left(1-y_{1,1}^{1,2}\right)+0,34375 y_{1,1}^{1,2}+0,25\left(1-y_{1,1}^{2,1}\right) \\
+0,15625 y_{1,1}^{2,1}+0,625\left(1-y_{1,2}^{2,2}\right)+0,125 y_{1,2}^{2,2}+0,34375\left(1-y_{1,1}^{3,2}\right)+0,15625 y_{1,1}^{3,2} \leq 2,5 \\
y_{1,2}^{1,1} \leq M x_{1}^{1,1,2}, y_{1,1}^{1,2} \leq M x_{1}^{1,2,1}, y_{1,1}^{2,1} \leq M x_{1}^{2,1,2}, y_{1,2}^{2,2} \leq M x_{1}^{2,2,2}, y_{1,1}^{3,2} \leq M x_{1}^{3,2,2} \\
0,375 x_{1}^{1,1,2}+0,375 x_{1}^{1,2,1}+0,875 x_{1}^{2,1,2}+0,875 x_{1}^{2,2,2}+x_{1}^{3,2,2} \leq 2,5
\end{gathered}
$$

Solving model (16),(20),(22),(23) we get the information that measures $M_{1}^{1,1,2}$ and $M_{1}^{2,1,1}$ should be applied, at the cost of 80 . However, if we change the weight of the risk attributes in the last column of Table 2, e.g. if the probability gets the weight $\mathrm{VH}$, there is no solution and we have to accept a higher risk level: $R L=(3,4,4)$. In that case the cheapest way of attaining this risk level would be the application of measures $M_{1}^{1,2,1}$ and $M_{1}^{3,2,1}$. 


\section{Conclusions}

A new method of selecting risk countermeasures to attain the desired risk level has been proposed. It allows to choose risk countermeasures taking into account such elements as various risk attributes and various weights assigned to them by the decision maker, various risk levels (the ones closer to the project, where we have more influence, and the ones further away), the monetary cost linked to the countermeasures as well as the immeasurable effort their application implies. The information required from the decision maker may be given in linguistic terms, modeled by fuzzy numbers. A real world example has been presented too.

Further research is necessary to verify the approach in other real world projects as well as to support the decision maker in the choice of the definition of fuzzy inequalities, which may be interpreted in many ways. The choice of the interpretation of fuzzy constraints has to correspond to the preferences of the decision maker, otherwise the model will not lead to a satisfying solution.

\section{Bibliography}

[1] Courtot H. (1988), La gestion des risques dans les projets, Ed. Economica, Paris.

[2] Camilieri E. (2011), Project Success: Critical Factors and Behaviours, Gower Publishing Company.

[3] Liu X. (2001), Measuring the satisfaction of constraints in fuzzy linear programming, Fuzzy Sets and Systems, 122(2): 263-275.

[4] Mandal S., Maiti J. (2013), Risk analysis using FMEA: Fuzzy similarity value and possibility theory based approach, Expert Systems with Applications, 41(7): 3527-3537.

[5] Meyer A., Zimmermann, H. (2011); Applications of fuzzy technology in business intelligence. International Journal of Computers Communications \& Control, 6(3): 428-441.

[6] Norman T.L. (2010), Risk Analysis and Security Countermeasure Selection, CRC Press.

[7] Shang K., Hossen Z. (2013); Research Paper: Applying Fuzzy Logic to Risk Assessment and Decision-Making, Casualty Actuarial Society, Canadian Institute of Actuaries, Society of Actuaries, 1-59.

[8] Skorupka D. (2012), Method of Construction Projects Risk Assessment, Lambert Academic Publishing.

[9] Wang Y.-M., Chin K.-S., Poon G. K. K., Yang J.-B. (2009), Risk evaluation in failure mode using fuzzy weighted geometric mean, Expert Systems with Applications, 1195-1207.

[10] Zhang Y., Zhi-Ping P. (2013); An optimization method for selecting project risk response strategies, International Journal of Project Management, 32(3); 412-422. 